\title{
Association between Serum Anti-glycopeptidolipid- core IgA Antibody Titers and Clinical Characteristics of Mycobacterium Avium Complex Pulmonary Disease
}

\section{Norio Kodaka}

Toho University Ohashi Medical Center

Chihiro Nakano

Toho University Ohashi Medical Center

\section{Takeshi Oshio}

Toho University Ohashi Medical Center

\section{Kayo Watanabe}

Toho University Ohashi Medical Center

\section{Kumiko Niitsuma}

Toho University Ohashi Medical Center

Chisato Imaizumi

Toho University Ohashi Medical Center

Nagashige Shimada

Toho University Ohashi Medical Center

\section{Hirotsugu Morita}

Toho University Ohashi Medical Center

Hiroto Matsuse ( $\nabla$ hiroto.matsuse@med.toho-u.ac.jp )

Toho University Ohashi Medical Center

\section{Research Article}

Keywords: Mycobacterium avium complex pulmonary disease (MAC-PD), anti-glycopeptidolipid (GPL), antibodies, nontuberculous mycobacterial diseases

Posted Date: March 12th, 2021

DOI: https://doi.org/10.21203/rs.3.rs-283326/v1

License: (9) This work is licensed under a Creative Commons Attribution 4.0 International License. Read Full License 
Version of Record: A version of this preprint was published at International Journal of Infectious Diseases on August 1st, 2021. See the published version at https://doi.org/10.1016/j.ijid.2021.06.042. 


\section{Abstract}

Mycobacterium avium complex pulmonary disease (MAC-PD) can be serologically diagnosed according to the presence of anti-glycopeptidolipid (GPL)-core IgA antibodies. However, few studies have examined the association between serum anti-GPL-core $\lg A$ antibody titers and the clinical characteristics of patients with MAC-PD. From April 2014 to June 2019, we determined the level of anti-GPL-core IgA antibodies in 489 MAC-PD patients at our institute. Of them, 89 patients fulfilled the criteria of the American Thoracic Society and the Infectious Diseases Society of America statement on the diagnosis, treatment, and prevention of nontuberculous mycobacterial diseases. These patients were divided into antibody-positive ( $n=59)$ or -negative $(n=30)$ groups according to their serum anti-GPL-core IgA antibody results. Additionally, the positive antibody group was further divided into a strong positive group $(n=27)$ and a weak positive group $(n=32)$, and their clinical characteristics were retrospectively compared. Disease progression requiring treatment during the 12 months following diagnosis and extensive radiological findings were significantly abundant in the strong positive group compared with the weak positive group. Our findings revealed that serum anti-GPL-core IgA antibody titers are useful not only for diagnosing MAC-PD but also for predicting the risk of exacerbation.

\section{Introduction}

The number of patients with Mycobacterium avium complex pulmonary disease (MAC-PD) is increasing globally [1-6]. In particular, there is an increasing number of cases of women with MAC-PD with central granular shadows of the middle lobe of the lung. Therefore, knowledge pertaining to its diagnosis, treatment, and management is essential for respiratory physicians. MAC-PD is the most frequently occurring nontuberculous mycobacteria (NTM) pulmonary disease [7], and it is often encountered in clinical practice. MAC-PD may have poor airway symptoms, and because the causative bacteria are not always detected in sputum samples, bronchoscopy may be essential for diagnosis. Furthermore, it may be difficult to detect bacteria by bronchoscopy, and some patients are hesitant to undergo bronchoscopy. Additionally, because MAC exists universally in soil, natural water, and tap water, it is difficult to distinguish between colonization and contamination [8].

MAC-PD is unlikely in patients who have a single positive sputum culture during the initial evaluation [911], but the rate of MAC-PD can be as high as $98 \%$ in those with $\geq 2$ positive sputum cultures [9].

Therefore, a diagnosis of MAC-PD should be made if MAC is identified at least twice in sputum samples or once in bronchial washings. However, although relatively advanced MAC-PD easily meets the diagnostic criteria, sputum collection is not possible with asymptomatic patients, and a long period is required for a definitive diagnosis. In such cases, the level of serum anti-glycopeptidolipid (GPL)-core IgA is used for diagnosis and has been utilized in clinical practice in Japan since August 2011. The GPL core is part of the GPL antigen, which is found on the surface of the cell walls of MAC but not of Mycobacterium tuberculosis or Mycobacterium kansasii. It can be detected in the serum of patients with MAC infection using commercially available serodiagnostic kits that measure the antibody level. Several studies have reported a sensitivity of $58.6-85 \%$ and a specificity of $96.9-100 \%$ [12-17]. The presence of 
anti-GPL-core IgA antibody is useful as a tool for the auxiliary diagnosis of MAC-PD, but since it is only used for auxiliary diagnosis, there are few reports comparing the differences in antibody titers. Thus, we compared the clinical course of cases with antibody titers of $\geq 5.0 \mathrm{U} / \mathrm{mL}$ (strong positive), $0.7-5.0 \mathrm{U} / \mathrm{mL}$ (weak positive), and $<0.7 \mathrm{U} / \mathrm{mL}$ (negative).

\section{Results}

Table 1 shows the clinical characteristics of the 89 MAC-PD patients in our cohort, including the microbiological and radiological findings. Of them, two-thirds were positive for anti-GPL-core IgA antibodies. None of the patients were known to be seropositive for human immunodeficiency virus. The male sex, infection with $M$. intracellulare, a history of smoking, the presence of a cavitary lesion, a MACpositive sputum smear, and the administration of medications for MAC during the year following diagnosis were reported in approximately one-fifth of patients, respectively. The median body mass index (BMI) was toward the lower range of normal, and the median number of abnormal lung zones was four. More than three-quarters of the patients had comorbidities (Table 1). 
Table 1

Baseline characteristics of MAC-PD patients with anti-GPL-core $\lg A$ antibody measured $(N=89)$

\section{Characteristic}

Age (years)

Female (\%)

$\operatorname{BMI}\left(\mathrm{kg} / \mathrm{m}^{2}\right)$

$\operatorname{TP}(\mathrm{g} / \mathrm{dL})$

$\operatorname{ALB}(\mathrm{g} / \mathrm{dL})$

$\mathrm{CRP}(\mathrm{mg} / \mathrm{dL})$

$\lg G(\mathrm{mg} / \mathrm{dL})$

$\lg \mathrm{E}(\mathrm{IU} / \mathrm{mL})$

Smoking history

Previous tuberculosis

Comorbidity

Bloody sputum

Positive MAC smear

Mycobacterium intracellulare

Cavitary lesion

Zones with radiological findings

Anti-GPL-core IgA antibody(strong/weak/negative)

Received treatment for MAC within 1 year of diagnosis

Data are expressed as medians (interquartile range) or numbers (\%).

ALB, serum albumin; BMI, body mass index; $\mathrm{CRP}$, serum C-reactive protein; GPL, glycopeptidolipid;
MAC, Mycobacterium avium complex; MAC-PD, Mycobacterium avium complex pulmonary disease;
TP, serum total protein

ALB, serum albumin; BMI, body mass index; $\mathrm{CRP}$, serum C-reactive protein; GPL, glycopeptidolipid;
MAC, Mycobacterium avium complex; MAC-PD, Mycobacterium avium complex pulmonary disease;
TP, serum total protein

ALB, serum albumin; BMI, body mass index; CRP, serum C-reactive protein; GPL, glycopeptidolipid;
MAC, Mycobacterium avium complex; MAC-PD, Mycobacterium avium complex pulmonary disease;
TP, serum total protein

\section{Baseline value}

$75(66-80)$

$71(79.8)$

$19.1(17.9-21.5)$

$7.4(7.0-7.9)$

$3.8(3.15-4.05)$

$0.14(0.04-1.21)$

$1380(1,227-1,555)$

70.8 (17.5-189.8)

19 (21.3\%)

$7(7.9 \%)$

$69(77.5 \%)$

$13(14.6 \%)$

18 (20.2\%)

$17(19.1 \%)$

$17(19.1 \%)$

$4(3-4)$

$27 / 32 / 30$

17 (19.1\%)

Regarding disease progression requiring treatment, extensive radiological findings were significantly abundant in the strong positive group compared with the weak positive group. Positive acid-fast bacilli (AFB) smears were significantly abundant in the strong positive group compared with the antibodynegative group. Those with weak positive antibody titers did not significantly differ from those in the antibody-negative group (Table 2). 
Table 2

Comparison of patient characteristics by anti-GPL-core $\lg A$ antibody titers $(N=89)$

\begin{tabular}{|c|c|c|c|c|}
\hline Characteristics & $\begin{array}{l}\text { Strong positive } \\
(n=27)\end{array}$ & $\begin{array}{l}\text { Weak positive } \\
(n=32)\end{array}$ & $\begin{array}{l}\text { Negative }(n \\
=30)\end{array}$ & $\begin{array}{l}P \text { - } \\
\text { value }^{\dagger}\end{array}$ \\
\hline Age, years & $73(62.5-77.5)$ & $\begin{array}{l}77.5(69.75- \\
82.25)\end{array}$ & $\begin{array}{l}74(67.5- \\
79.0)\end{array}$ & 0.20 \\
\hline Sex (female, \%) & $23(85.2 \%)$ & $26(81.3 \%)$ & $22(73.3 \%)$ & 0.53 \\
\hline $\mathrm{BMI}\left(\mathrm{kg} / \mathrm{m}^{2}\right)$ & $\begin{array}{l}18.7(17.2- \\
19.8)\end{array}$ & $\begin{array}{l}20.5(18.1- \\
22.4)\end{array}$ & $\begin{array}{l}19.4(16.6- \\
21.0)\end{array}$ & 0.13 \\
\hline $\mathrm{TP}(\mathrm{g} / \mathrm{dL})$ & $7.4(6.9-7.9)$ & $7.5(7.2-7.9)$ & $\begin{array}{l}7.4(6.9- \\
7.8)\end{array}$ & 0.80 \\
\hline ALB (g/dL) & $3.7(3.4-4.0)$ & $3.9(3.6-4.2)$ & $\begin{array}{l}3.6(3.0- \\
4.0)\end{array}$ & 0.16 \\
\hline CRP (mg/dL) & $\begin{array}{l}0.09(0.03- \\
0.61)\end{array}$ & $\begin{array}{l}0.09(0.03- \\
0.90)\end{array}$ & $\begin{array}{l}0.29(0.11- \\
2.6)\end{array}$ & 0.12 \\
\hline $\lg G(\mathrm{mg} / \mathrm{dL})$ & $\begin{array}{l}1336(1233- \\
1507)\end{array}$ & $\begin{array}{l}1387(1242- \\
1437)\end{array}$ & $\begin{array}{l}1457 \\
(1144- \\
1609)\end{array}$ & 0.66 \\
\hline $\operatorname{lgE}(\mathrm{IU} / \mathrm{mL})$ & $\begin{array}{l}75.3(19.9- \\
194.9)\end{array}$ & $37.4(17.3-84)$ & $\begin{array}{l}73.5(20.0- \\
293)\end{array}$ & 0.52 \\
\hline Smoking history & $6(22.2 \%)$ & $3(9.4 \%)$ & $10(30.3 \%)$ & 0.07 \\
\hline Previous tuberculosis & $1(3.7 \%)$ & $3(9.4 \%)$ & $3(10.0 \%)$ & 0.63 \\
\hline Comorbidity & $21(77.8 \%)$ & $27(84.4 \%)$ & $21(70.0 \%)$ & 0.40 \\
\hline Bloody sputum & $6(22.2 \%)$ & $6(18.8 \%)$ & $1(3.3 \%)$ & 0.09 \\
\hline Positive MAC smear & $11(40.7 \%)^{\ddagger}$ & $6(18.8 \%)$ & $1(3.3 \%)$ & $0.002^{*}$ \\
\hline Mycobacterium intracellulare & $2(7.4 \%)$ & $9(28.1 \%)$ & $6(20.0 \%)$ & 0.13 \\
\hline Cavitary lesion & $6(22.2 \%)$ & $7(21.9 \%)$ & $4(13.3 \%)$ & 0.62 \\
\hline Zones with radiological findings & $4(3-5)$ & $3(2-4)$ & $3(2-4)$ & $0.03^{*}$ \\
\hline Anti-GPL-core IgA antibody titers & $16(9.5-32.1)$ & $\begin{array}{l}2.1(1.28- \\
3.1)^{\ddagger}\end{array}$ & $\leq 0.7$ & $<0.001^{*}$ \\
\hline $\begin{array}{l}\text { Received treatment for MAC within } 1 \\
\text { year of diagnosis }\end{array}$ & $10(37.0 \%)$ & $4(12.5 \%)$ & $3(10.0 \%)$ & $0.02^{*}$ \\
\hline \multicolumn{5}{|c|}{ Data are expressed as medians (interquartile range) or numbers (\%). } \\
\hline
\end{tabular}




\begin{tabular}{|c|c|c|c|c|}
\hline Characteristics & $\begin{array}{l}\text { Strong positive } \\
(n=27)\end{array}$ & $\begin{array}{l}\text { Weak positive } \\
(n=32)\end{array}$ & $\begin{array}{l}\text { Negative ( } n \\
=30)\end{array}$ & $\begin{array}{l}\boldsymbol{P} \\
\text { value }^{\dagger}\end{array}$ \\
\hline \multicolumn{5}{|c|}{$\dagger P$-values are for all comparisons. } \\
\hline \multicolumn{5}{|l|}{${ }^{*} P<0.05$} \\
\hline${ }^{9} P<0.05$ for ant & veak titers vs. ant & SPL-core IgA ant & ody-negative. & \\
\hline
\end{tabular}

\section{Discussion}

To our knowledge, this is the first report to describe the differences in clinical characteristics using an anti-GPL-core antibody titer threshold value of $5.0 \mathrm{U} / \mathrm{mL}$. We set the threshold of the antibody-positive group to $5.0 \mathrm{U} / \mathrm{mL}$, according to a previous report where the mean antibody titer that was considered the most reliable for MAC-PD diagnosis via bronchoscopy was $5.0 \mathrm{U} / \mathrm{mL}$ [18]. Among the antibody-positive MAC-PD patients in our study, the median value of anti-MAC antibodies was $4.5 \mathrm{U} / \mathrm{mL}$, but considering the application to actual clinical practice and an objective evaluation, we selected $5.0 \mathrm{U} / \mathrm{mL}$ as the threshold between the strong positive and weak positive groups in our study.

In our study, $66.3 \%$ of the patients newly diagnosed with MAC-PD were positive for anti-GPL-core IgA antibodies, which is comparable to the sensitivity reported in a previous review [19]. Furthermore, the clinical characteristics of the patients with MAC-PD in our study were consistent with those in previous reports (i.e., the patients were predominantly slim women with nodular bronchiectatic disease) $[1-6,8,20$, 21].

Kitada et al. reported that the antibody titers reflect the disease activity to some extent $[13,22,23]$ and found a weak correlation between the antibody levels and the extent of the disease on chest computed tomography (CT) $[12,24]$. Similarly, we found that both disease progression requiring treatment and extensive radiological findings were significantly abundant in the strong positive antibody group in our study cohort. However, there was no significant difference in the presence or absence of cavitary lesions. Thus, a strong positive anti-GPL-core IgA antibody result was associated with higher radiological scores for infiltration, although no difference was observed in cavitary lesions between patients with strong positive and weak positive results. This correlates with another study showing that the anti-GPL-core IgA antibody titer was not associated with the extent of disease in the fibrocavitary disease phenotype [22].

We found that a positive AFB smear in the strong positive group was significantly more abundant than in the antibody-negative group and slightly more abundant than in the weak positive group $(P=0.053)$. Extensive radiological findings $[23,25]$ and an AFB-positive sputum smear $[8,25,26]$ have been reported as exacerbating factors of MAC-PD, which increases the likelihood that a high antibody titer is an exacerbating factor. 
However, the anti-GPL-core IgA antibody titer in patients with severe MAC-PD was sometimes low in our study, probably due to differences in individual immunity and immune response ability, but no significant difference was found in serum total protein, serum albumin, IgG, and IgE levels in our study. A lower BMI was considered an exacerbating factor in MAC-PD patients in previous studies [27, 28]. In our study, although many patients with MAC-PD were thin on average, there was no significant difference in BMI between the three groups.

In our study, M. intracellulare was found in $18.6 \%$ of patients with MAC-PD, and the infection rate was similar to other reports in Japan [29]. Regarding MAC species differentiation, some studies reported that patients with $M$. intracellulare infection experienced more frequent exacerbations than those with $M$. avium infection [30], but others reported that patients with $M$. intracellulare infection experienced a lower rate of recurrence than those with $M$. avium infection [31].Thus, the difference in the prognosis between $M$. avium and $M$. intracellulare infections remains uncertain. In our study, there was no significant relationship between the prognosis and the MAC species in all three groups. Additionally, there was no significant difference in clinical features between the MAC-PD patients with weak positive anti-GPL-core $\lg A$ antibodies and those who were antibody-negative. Thus, MAC-PD patients with weak positive antibody titers were considered to be of no clinical importance in our study other than in the diagnostic method that was used.

\section{Limitations}

Our study has several limitations. First, because the sample size was small, the number of MAC-PD patients may have been underestimated since patients who were not diagnosed according to the American Thoracic Society (ATS) and the Infectious Disease Society of America (IDSA) guidelines were excluded from the analyses. Therefore, in reality, factors with clinical significance may have proven insignificant in the analyses due to reduced statistical power. Second, because data collection was retrospective, sputum cultures, laboratory data, and CT scans were performed according to clinical practice rather than according to strict schedules. Finally, the timing of the treatment was dependent on the decision of the attending physician.

\section{Conclusions}

Our study shows that anti-GPL-core IgA antibody titers $>5.0 \mathrm{U} / \mathrm{mL}$ can be used to both diagnose MAC-PD and to predict the risk of exacerbation. However, patients with weak positive antibody titers are not considered clinically significant other than in diagnostic methods. Although the anti-GPL-core IgA antibody is a qualitative test for MAC-PD, it can be useful as a quantitative test for MAC-PD severity.

\section{Methods}

\section{Study Population and Design}


We retrospectively reviewed all patients seen in our hospital in Tokyo, Japan, from April 2014 to June 2019. There were 481 patients who underwent serologic testing for anti-GPL-core IgA antibodies. Of them, 119 newly diagnosed NTM-PD patients fulfilled the criteria of the ATS and the IDSA statement on the diagnosis, treatment, and prevention of nontuberculous mycobacterial diseases [8,26]. Those with a past history of NTM disease, NTM other than MAC, and no abnormal shadow on chest CT were excluded, and the remaining 89 MAC-PD patients were enrolled in our study (Fig. 1). We divided the study patients into two groups according to anti-GPL-core IgA antibody results: an antibody-positive group $(n=59)$ and an antibody-negative group ( $n=30$, antibody titer $<0.7 \mathrm{U} / \mathrm{mL}$ ). Then, the antibody-positive group was further divided into a strong positive group $(n=27$, antibody titer $\geq 5 \mathrm{U} / \mathrm{mL})$ and a weak positive group $(n=32$, $5.0<$ antibody titer $\geq 0.7 \mathrm{U} / \mathrm{mL}$ ). The antibody titers and the clinical characteristics of the patients, including age, sex, laboratory data, a past history of tuberculosis, comorbidities, and radiological findings, were retrospectively compared. Additionally, we investigated whether treatment was required for up to 1 year after diagnosis to determine if anti-GPL-core IgA antibody titers were associated with disease progression.

\section{Ethics Approval and Consent to Participate}

All methods were carried out in accordance with the declaration of Helsinki. The need of informed consent was waived by the Ethics Committee of Toho University Ohashi Medical Center due to the retrospective nature of the study. All protocols were approved by the Ethics Committee of Toho University Ohashi Medical Center (approval no. H20004).

\section{Serological Determination of Anti-GPL-core IgA Antibody Titers}

The titer of serum anti-GPL-core IgA antibodies was determined using a Capilia MAC Ab ELISA serodiagnostic kit (TAUNS Laboratories, Inc., Shizuoka, Japan). The cutoff value was defined as 0.7 $\mathrm{U} / \mathrm{mL}$, according to the manufacturer's instructions [22]. The test can be performed using a small amount of serum, and the turnaround time is approximately 4 hours [32].

\section{Microbiological Examination}

AFB were cultured in mycobacteria growth indicator tubes using sputum samples or bronchial washings obtained by bronchoscopy. The sputum samples were obtained on two or more occasions after the initial presentation. The diagnosis of MAC-PD was made when MAC was identified in sputum at least twice or once in bronchial washings. MAC was confirmed when cultures were positive for AFB, and the cultured AFB were subsequently confirmed as MAC by polymerase chain reaction. 


\section{Radiological Examination and Measurements}

High-resolution chest CT findings were classified as either showing or not showing a cavitary lesion. Additionally, chest CT findings at the time of initial diagnosis were scored, as previously described [33]. Briefly, the lung fields were divided into six lobes based on anatomical structures: right upper, right middle, right lower, left upper (S1+2 and S3), left lingular (S4 and S5), and left lower lobes. We assessed whether, at the time of diagnosis, each lung lobe had a shadow, such as cavities, bronchiectasis, small nodules, consolidations, or atelectasis.

\section{Statistical Analysis}

The patients' characteristics are presented as medians (interquartile range). Numerical data are expressed as numbers (\%). Intergroup differences (anti-GPL-core IgA antibody strong positive group vs. weak positive group vs. negative group) were compared using the Kruskal-Wallis test for numerical variables and the chi-square test or Fisher exact test for categorical variables where appropriate. All analyses were performed using SPSS Statistical software v22.0 (IBM Japan, Tokyo, Japan). P-values < 0.05 were considered significant.

\section{Declarations}

\section{Author contributions}

N.K. collected the data, analyzed the data, created the tables, designed the study, and wrote the manuscript. C.N., T.O., K.W., K.N., C.I., N.S., H.Mo., and H.Ma. collected the data. H.Ma. critically revised the manuscript. All authors read and approved the final manuscript.

\section{Additional information}

\section{Data availability statement}

The data that support the findings of this study are available on request from the corresponding author. The data are not publicly available due to ethical restrictions.

\section{Competing interests}

The authors declare no competing interests.

\section{References}


1. Prevots, D. R. \& Marras, T. K. Epidemiology of human pulmonary infection with nontuberculous mycobacteria: a review. Clin. Chest Med. 36, 13-34 (2015).

2. Tan, Y. et al. Epidemiology of pulmonary disease due to nontuberculous mycobacteria in Southern China, 2013-2016. BMC Pulm. Med. 18, 168 (2018).

3. Furuuchi, K. et al. Interrelational changes in the epidemiology and clinical features of nontuberculous mycobacterial pulmonary disease and tuberculosis in a referral hospital in Japan. Respir. Med. 152, 74-80 (2019).

4. Kendall, B. A. \& Winthrop, K. L. Update on the epidemiology of pulmonary nontuberculous mycobacterial infections. Semin. Respir. Crit. Care Med. 34, 87-94 (2013).

5. Hoefsloot, W. et al. The geographic diversity of nontuberculous mycobacteria isolated from pulmonary samples: an NTM-NET collaborative study. Eur. Respir. J. 42, 1604-1613 (2013).

6. Simons, S. et al. Nontuberculous mycobacteria in respiratory tract infections, eastern Asia. Infect. Dis. 17, 343-349 (2011).

7. Hoefsloot, W. et al. The geographic diversity of nontuberculous mycobacteria isolated from pulmonary samples: an NTM-NET collaborative study. Eur. Respir. J. 42, 1604-1613 (2013).

8. Daley, C. L. et al. Treatment of nontuberculous mycobacterial pulmonary disease: an Official ATS/ERS/ESCMID/IDSA Clinical Practice Guideline: Executive Summary. Infect. Dis. 71, e31-e36 (2020).

9. Tsukamura, M. Diagnosis of disease caused by Mycobacterium avium Chest. 99, 667-669 (1991).

10. Koh, W. J. et al. Clinical significance of a single isolation of pathogenic nontuberculous mycobacteria from sputum specimens. Diagn. Microbiol. Infect. Dis. 75, 225-226 (2013).

11. Lee, M. R. et al. Factors associated with subsequent nontuberculous mycobacterial lung disease in patients with a single sputum isolate on initial examination. Clin. Microbiol. Infect. 21,e1-7 (2015).

12. . Kitada, S. et al. Validation of a commercial serodiagnostic kit for diagnosing pulmonary Mycobacterium avium complex disease. Int. J. Tuberc. Lung Dis. 19, 97-103 (2015).

13. Numata, T. et al. Clinical efficacy of anti-glycopeptidolipid-core IgA test for diagnosing Mycobacterium avium complex infection in lung. Respirology. 20, 1277-1281 (2015).

14. Shu, C.-C. et al. Sero-diagnosis of Mycobacterium avium complex lung disease using serum immunoglobulin A antibody against glycopeptidolipid antigen in Taiwan. PLoS 8, e80473 (2013).

15. Kobashi, Y., Mouri, K., Obase, Y., Kato, S. \& Oka, M. Serological assay by use of glycopeptidolipid core antigen for Mycobacterium avium Scand. J. Infect. Dis. 45, 241-249 (2013).

16. Kitada, S. et al. Serodiagnosis of Mycobacterium avium complex pulmonary disease in the USA. Eur. Respir. J. 42, 454-460 (2013).

17. Jeong, B. H. et al. Serodiagnosis of Mycobacterium avium complex and Mycobacterium abscessus complex pulmonary disease by use of IgA antibodies to glycopeptidolipid core antigen. Clin. Microbiol. 51, 2747-2749 (2013). 
18. S. et al. Serodiagnosis of pulmonary disease due to Mycobacterium avium complex proven by bronchial wash culture. Chest. 138, 236-237 (2010).

19. Shibata, Y. et al. Diagnostic test accuracy of anti-glycopeptidolipid-core IgA antibodies for Mycobacterium avium complex pulmonary disease: systematic review and meta-analysis. Sci. Rep. 6, 29325 (2016).

20. Izumi K. et al. Epidemiology of adults and children treated for nontuberculous mycobacterial pulmonary disease in Japan. Ann. Am. Thorac. Soc. 16, 341-347 (2018).

21. Song, J. H., Kim, B. S., Kwak, N., Han, K., \& Yim, J.-J. Impact of body mass index on development of nontuberculous mycobacterial pulmonary disease. Eur. Respir. J. 57, 2000454 (2021).

22. Kitada, S. et al. Serodiagnosis of Mycobacterium avium-complex pulmonary disease using an enzyme immunoassay kit. J. Respir. Crit. Care Med. 177, 793-797 (2008).

23. Kitada, S. et al. Use of glycopeptidolipid core antigen for serodiagnosis of Mycobacterium avium complex pulmonary disease in immunocompetent patients. Clin. Diagn. Lab. Immunol. 12, 44-51 (2005).

24. Kitada, S. et al. Serological test and chest computed tomography findings in patients with Mycobacterium avium complex lung disease. Eur. Respir. J. 29, 1217-1223 (2007).

25. Hwang, J. A., Kim, S., Jo K.-W. \& Shim, T. S. Natural history of Mycobacterium avium complex lung disease in untreated patients with stable course. Eur. Respir. J. 49, 1600537 (2017).

26. Griffith, D. E. et al. An official ATS/IDSA statement: diagnosis, treatment, and prevention of nontuberculous mycobacterial diseases. J. Respir. Crit. Care Med. 175, 367-416 (2007).

27. Kim, S. J. et al. Risk factors for deterioration of nodular bronchiectatic Mycobacterium avium complex lung disease. J. Tuberc. Lung Dis. 18, 730-736 (2014).

28. Kitada, S. et al. Long-term radiographic outcome of nodular bronchiectatic Mycobacterium avium complex pulmonary disease. Int. J. Tuberc. Lung Dis. 16, 660-664 (2012).

29. Morimoto, K. et al. A laboratory-based analysis of nontuberculous mycobacterial lung disease in Japan from 2012 to 2013. Ann. Am. Thorac. Soc. 14, 49-56 (2017).

30. Koh, W. J. et al. Clinical significance of the differentiation between Mycobacterium avium and Mycobacterium intracellulare in M avium complex lung disease. 142, 1482-1488 (2012).

31. Boyle, D. P., Zembower, T. R., Reddy, S. \& Qi, C. Comparison of clinical features, virulence, and relapse among Mycobacterium avium complex species. J. Respir. Crit. Care Med. 191, 1310-1317 (2015).

32. Kitada S. Application of a commercial serodiagnostic kit that measures the serum antiglycopeptidolipid core IgA antibody in Mycobacterium avium complex pulmonary disease. Respir Investig. 57:410-414(2019).

33. Kodaka, N. et al. Predictors of radiological aggravations of pulmonary MAC disease. PLoS One. 15, e0237071 (2020).

\section{Figures}


Figure 1

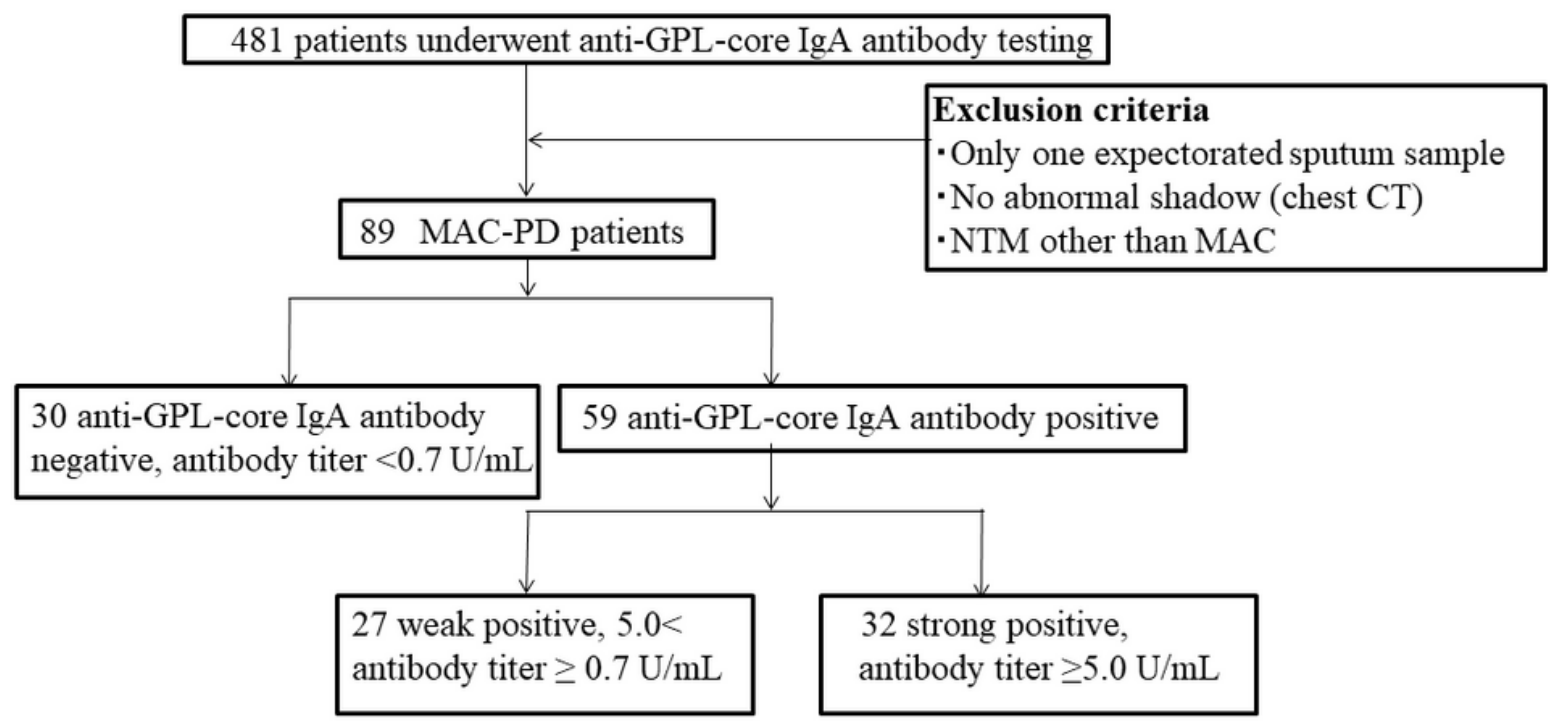

\section{Figure 1}

Flow chart of patients diagnosed with MAC pulmonary disease between April 2014 and June 2019 at our institution. MAC-PD, Mycobacterium avium complex pulmonary disease; CT, computed tomography; NTM, nontuberculous mycobacteria; MAC, Mycobacterium avium complex; GPL, glycopeptidolipid 